\title{
Clinical Analysis of the Predictors for Intravesical Recurrence After Laparoscopic Radical Nephroureterectomy in Japanese Patients
}

\author{
Satoshi Fukata*, Hideo Fukuhara, Shingo Ashida, Takashi Karashima, Keiji Inoue \\ Department of Urology, Kochi University School of Medicine, Nankoku, Kochi, Japan \\ Email address: \\ jm-fukatas@kochi-u.ac.jp (S. Fukata) \\ ${ }^{*}$ Corresponding author \\ To cite this article: \\ Satoshi Fukata, Hideo Fukuhara, Shingo Ashida, Takashi Karashima, Keiji Inoue. Clinical Analysis of the Predictors for Intravesical \\ Recurrence After Laparoscopic Radical Nephroureterectomy in Japanese Patients. International Journal of Clinical Urology. \\ Vol. 4, No. 1, 2020, pp. 6-12. doi: 10.11648/j.ijcu.20200401.12
}

Received: December 15, 2019; Accepted: January 9, 2020; Published: January 21, 2020

\begin{abstract}
Objectives: In this study, we evaluate the clinicopathological independent prognostic factors which to predict IVR in patients who underwent Laparoscopic-assisted radical nephroureterectomy (LRNU) for upper urinary tract carcinoma (UTUC). Methods: Between April 2008 to February 2019, we analyzed 100 japanese patients who were underwent LRNU and 92 patients who were underwent open radical nephroureterectomy (ORNU) at our institutions by retrospectively. The Patients characteristics factors, the clinicopathologic factors were collected. The intravesical recurrence free survival (IVRFS) were analyzed using the Kaplan-Meier method and Univariate and multivariate method by using the Cox proportional hazards regression models were performed to identify independent risk factors for IVR after LRNU. Results: In LRNU group, IVR was observed in 39 cases (39\%), and it was not significant difference compare with ORNU ( $\mathrm{P}: 0.36)$. Tumor location $(\mathrm{P}=0.002)$, Tumor size $(\mathrm{P}=0.001)$, preoperative urine cytology $(\mathrm{P}<0.0001)$, the pneumoperitoneum time $(\mathrm{P}$ : 0.0005$)$ and adjuvant chemotherapy ( $\mathrm{P}=0.019)$ showed significant association with postoperative IVR. In the multivariate Cox hazard models, the tumor location ( $\mathrm{P}=0.0003)$, positive preoperative urinary cytology $(\mathrm{P}=0.003)$, and absence of adjuvant chemotherapy $(\mathrm{P}=0.003)$ were independent risk factors for subsequent IVR. There were not associated with smoking, Brinkman index, hydronephrosis and ureterorenoscopy before RNU for IVR. Overal survival (OS) was not significant association for experience IVR ( $\mathrm{P}=0.15)$. Conclusion: In this study, LRNU was not shown to have a significantly higher IVR compared to ORNU. Patients with ureteral cancer and positive preoperative urinary cytology had a higher risk of IVR after LRNU. The adjuvant chemotherapy was significantly decreased the risk for postoperative IVR.
\end{abstract}

Keywords: Intravesical Recurrence, Laparoscopic Nephroureterectomy, Upper tract Urothelial Carcinoma

\section{Introduction}

Radical nephroureterectomy (RNU) is widely performed as a standard procedure for treatment of the UTUC. In recently, the opportunities of laparoscopic surgery have increased in the RNU with the development of laparoscopic techniques. As a result, surgical invasiveness is reduced, and RNU can be performed relatively safely even in cases with many complications or elderly patients. However, there are disadvantages to LRNU. One of them is the risk of postoperative IVR. Some studies have discussed clinicopathologic specific risk factors for prediction of IVR $[1,2]$. In Meta-analysis, laparoscopic procedure has been reported to be a significant risk factor for IVR [3]. The IVR after RNU often occurs with an approximately $15 \%-50 \%$ incidence [4]. However, the certain mechanism of IVR after LRNU has been unclear. In this study we evaluate the association of the predictive factors for postoperative IVR in japanese patients underwent LRNU.

\section{Patients and Methods}

\subsection{Patients}

From April 2008 to February 2019, we retrospectively identified a total of 192 UTUC patients who underwent either 
ORNU or LRNU. 100 patients who were underwent LRNU and 92 patients were ORNU were entried in this study. The patient background factors (age, gender, smoking history, Blickmann index), Preoperative clinico-pathological data (presence of hydronephrosis, Preoperative urinecytology and Preoperative ureterorenoscopy (URS)) were retrospectively collected. The tumor location was defined as the renal pelvis and/or upper, middle, lower ureter. The pathological factors in the resected specimen, tumor size, pathological $\mathrm{T}$ stage (pT), vascular invasion, lymphatic vessel invasion, and presence of resection margin were examined. Perioperative clinical data including of total surgical time, pneumoperitoneum time and blood loss were examined. LRNU was performed according to the standard procedure. The kidney and upper-middle ureter are resected and liberated laparoscopically (retroperitoneal approach), and as soon as possible, the distal ureter is clipped, and then we performed that peeling and excavation of the lower ureter and bladder cuff resection and extracted the kidney and ureter en bloc by pararectal incision. The bladder cuff resection was performed through the extravesical technique of the ureteral orifice. There were no cases in which an immediately intravesical therapy for the IVR prevention. Lymphadenectomy was not performed regularly, except suspiciously enlarged lymph nodes. The infusing pressurized $\mathrm{CO}_{2}$ gas was difined with a standard pressure of $8 \mathrm{mmHg}$ to maximum pressure of $12 \mathrm{mmHg}$. Preoperative urine cytology was evaluated using voided samples. Preoperative diagnosis of hydronephrosis was made using computed tomography (CT). Preoperative URS was performed within 2 months before LRNU. No patients underwent neoadjuvant chemotherapy. Adjuvant chemotherapy was performed on the basis of pathological diagnosis (pT1 or above) or positive vascular invasion case.

\subsection{Follow-up Regimen}

Cystoscopy was performed on all patients and urine cytology every 3 months for the first 3 years, every 6 months after 3 years from LRNU. IVR was diagnosed as pathologic proof through transurethral biopsy and/or resection.

\subsection{Factors to Consider, Statistical Methods}

Association between clinic-pathological factors and IVR was examined using statistical analysis (Wilcoxon signed-rank test or Fisher's exact test for variables). Intravesical recurrence-free survival (IVRFS), and overall survival (OS) after LRNU were used the Kaplan-Meier method. The prognostic effect of clinic-pathological factors were estimated by Univariate and multivariate method by using the Cox proportional hazards regression models. The strength of individual variables were assessed using the hazard ratios (HRs) with 95\% confidence intervals. We performed statistical analysis using $\mathrm{JMP}^{\circledR}$ 8.0 (SAS Institute Inc., Cary, NC, USA) and defined statistical significance as $\mathrm{P}$ value $<0.05$.

\section{Results}

\subsection{Patients Background}

A total of $66(34.3 \%)$ patients experienced IVR. The median follow-up time was 19.4 months (range, 1-219 months), and the median interval between LRNU and the first IVR was 13.6months (range 1-108 months).

\subsection{The Comparison Between ORNU and LRNU of IVR}

Table 1. Shows the background of 92 ORNU patients and $100 \mathrm{LRNU}$ patients registered in this study. There was no significant difference of postoperative IVR rate compare with LRNU and ORNU (P: 0.36).

\begin{tabular}{|c|c|c|c|}
\hline & ORNU & LRNU & $P$ value \\
\hline $\mathrm{n}$ & 92 & 100 & \\
\hline Follow-up period, day & 1450 & 1218 & 0.53 \\
\hline age, y & $66(42-88)$ & $73(50-92)$ & 0.0002 \\
\hline Gender (Male/Female) & $65 / 27$ & $73 / 27$ & 0.74 \\
\hline Laterality (left/right) & $43 / 49$ & $54 / 46$ & 0.26 \\
\hline Tumor lacation & & & 0.01 \\
\hline Renal pelvis & 51 & 40 & \\
\hline Upper ureter & 10 & 24 & \\
\hline Middle ureter & 8 & 21 & \\
\hline Lower ureter & 17 & 13 & \\
\hline multiple & 6 & 2 & \\
\hline $\begin{array}{l}\text { History of the Bladder cancer } \\
\text { Tumor grade }\end{array}$ & $11(12 \%)$ & $21(21 \%)$ & 0.09 \\
\hline low & 7 & 2 & \\
\hline high & 83 & 98 & \\
\hline unknouwn & 2 & 0 & \\
\hline Pathologic T stage & & & $<0.0001$ \\
\hline $\mathrm{pTa}$ & 0 & 21 & \\
\hline pT1 & 18 & 20 & \\
\hline pT2 & 20 & 19 & \\
\hline pT3 & 30 & 31 & \\
\hline pT4 & 10 & 2 & \\
\hline pTis & 14 & 7 & \\
\hline Vascular invsion & $28(34 \%)$ & $17(17 \%)$ & 0.01 \\
\hline Lymphovascular invasion & $26(30.5 \%)$ & $22(22 \%)$ & 0.23 \\
\hline Pthologic N + & $9(10 \%)$ & $3(3 \%)$ & 0.04 \\
\hline Ajuvant chemotherapy & & & 0.31 \\
\hline No & 43 & 56 & \\
\hline yes & 49 & 44 & \\
\hline Intra vesical reccurence & $28(30 \%)$ & $38(38 \%)$ & 0.36 \\
\hline
\end{tabular}

ORNU=open radical nephroureterectomy.

$\mathrm{LRNU}=$ laparoscopic radical nephroureterectomy.

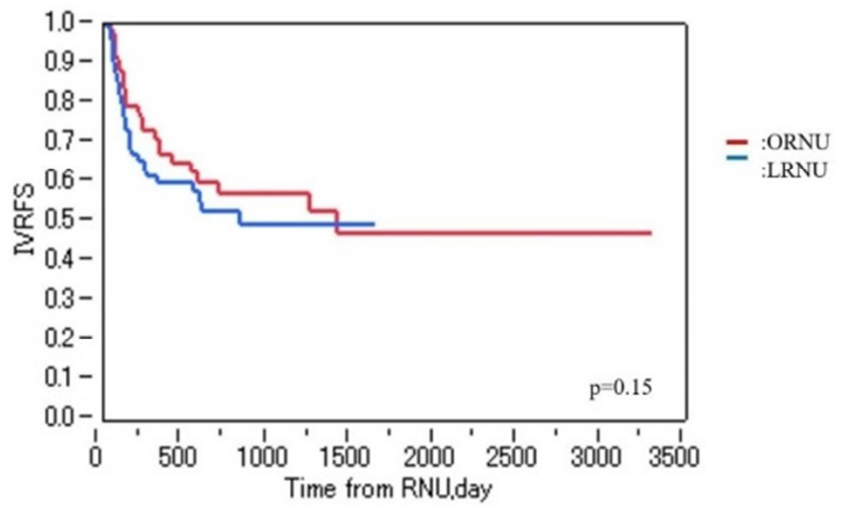

Figure 1. Showed that Kaplan-Meier curve of IVRFS in ORNU vS LRNU. There was no significant difference of IVR rate between the both groups $(P=0.15)$. 


\subsection{The Association Between Clinical Factors and IVR Rate in LRNU Patients}

Table 2. Was shows the clinic-pathological factors and operative outcome of 100 LRNU patients. A total 39 (39\%) patients experienced IVR. There was no significant association for IVR in gender, age and previous history of bladder cancer. However, there was significant association in tumor location ( $P=0.0002)$, smoking history $(P=0.03)$, Blickmann index $(P=0.01)$, hydronephrosis $(P=0.007)$ and Preoperative URS $(P=0.002)$

\begin{tabular}{|c|c|c|c|c|}
\hline Cgaracterristics & total no & $\begin{array}{l}\text { Negative } \\
\text { IVR (\%) }\end{array}$ & $\begin{array}{l}\text { Positive } \\
\text { IVR (\%) }\end{array}$ & $P$ value \\
\hline $\mathrm{n}$ & 100 & 61 & 39 & \\
\hline Age, y & & $73(55-92)$ & $72(50-86)$ & 0.65 \\
\hline Gender & & & & 0.64 \\
\hline male & 73 & $43(70.5)$ & $29(74.3)$ & \\
\hline female & 27 & $18(29.5)$ & $9(25.7)$ & \\
\hline Tumor location & & & & 0.002 \\
\hline renal pelvis & 50 & $36(59)$ & $14(35.9)$ & \\
\hline upper ureter & 15 & $9(14.8)$ & $6(15.4)$ & \\
\hline middle - & 14 & $11(18)$ & $3(7.7)$ & \\
\hline lower - & 19 & $5(8.2)$ & $14(35.9)$ & \\
\hline multiple & 2 & 0 & $2(5.1)$ & \\
\hline $\begin{array}{l}\text { History of Bladder } \\
\text { cancer }\end{array}$ & & & & 0.62 \\
\hline no & 79 & $50(81.9)$ & $30(76.9)$ & \\
\hline yes & 21 & $12(18.1)$ & $9(25.7)$ & \\
\hline $\begin{array}{l}\text { Preoperative urine } \\
\text { cytology }\end{array}$ & & & & $<0.0001$ \\
\hline negative & 53 & $42(68.8)$ & $11(28.2)$ & \\
\hline positive & 47 & $19(31.2)$ & $28(71.8)$ & \\
\hline Multifocality & & & & 0.12 \\
\hline negative & 87 & $57(93.4)$ & $30(76.9)$ & \\
\hline positive & 13 & $4(6.6)$ & $9(23.1)$ & \\
\hline Tumor size, mm & & 27 & 39 & 0.001 \\
\hline Pathologic T stage & & & & 0.01 \\
\hline $\mathrm{pTa}$ & 14 & $13(21.3)$ & $1(2.5)$ & \\
\hline pT1 & 26 & $17(27.8)$ & $9(23.1)$ & \\
\hline $\mathrm{pT} 2$ & 26 & $16(19.1)$ & $10(25.6)$ & \\
\hline pT3 & 25 & $9(14.7)$ & $16(41)$ & \\
\hline pT4 & 2 & $2(3.3)$ & 0 & \\
\hline pTis & 7 & $4(6.6)$ & $3(7.7)$ & \\
\hline Concomitant CIS & & & & 0.8 \\
\hline negative & 79 & 48 (78.7) & $31(79.5)$ & \\
\hline positive & 21 & $13(21.3)$ & $8(20.5)$ & \\
\hline Vascular invasion & & & & 0.67 \\
\hline negative & 82 & $51(83.6)$ & 33 (84.6) & \\
\hline positive & 17 & $10(16.4)$ & $6(15.4)$ & \\
\hline $\begin{array}{l}\text { Lymphovascular } \\
\text { invasion }\end{array}$ & & & & 0.62 \\
\hline negative & 78 & $48(78.7)$ & $30(76.9)$ & \\
\hline positive & 22 & $13(21.3)$ & $9(23.1)$ & \\
\hline Pathologic N stage & & & & 0.28 \\
\hline pNo & 97 & $58(95.1)$ & $39(100)$ & \\
\hline $\mathrm{pN} 1-2$ & 3 & $3(4.9)$ & 0 & \\
\hline Resection margin & & & & 0.7 \\
\hline negative & 86 & 53 (86.9) & $33(84.6)$ & \\
\hline positive & 8 & $6(9.8)$ & $2(5.1)$ & \\
\hline unknouwn & 6 & $2(3.3)$ & $4(10.3)$ & \\
\hline $\begin{array}{l}\text { Total operation } \\
\text { time (min, med) }\end{array}$ & & 370 & 396 & 0.25 \\
\hline $\begin{array}{l}\text { pneumoperitoneum } \\
\text { time (min, med) }\end{array}$ & & 197 & 237 & 0.0005 \\
\hline $\begin{array}{l}\text { Blood loss (ml, } \\
\text { med) }\end{array}$ & & 197 & 275 & 0.26 \\
\hline $\begin{array}{l}\text { Adjuvant } \\
\text { chemotherapy }\end{array}$ & & & & 0.019 \\
\hline no & 60 & 31 & 29 & \\
\hline
\end{tabular}

\begin{tabular}{|c|c|c|c|c|}
\hline Cgaracterristics & total no & $\begin{array}{l}\text { Negative } \\
\text { IVR (\%) }\end{array}$ & $\begin{array}{l}\text { Positive } \\
\text { IVR (\%) }\end{array}$ & $P$ value \\
\hline yes & 40 & 30 & 10 & \multirow{4}{*}{0.034} \\
\hline Smoking history & & & & \\
\hline no & 41 & 30 & 11 & \\
\hline yes & 59 & 31 & 28 & \\
\hline Blickmann index & & 373 & 716 & 0.01 \\
\hline $\begin{array}{l}\text { Previous } \\
\text { hydronephrosis }\end{array}$ & & & & 0.007 \\
\hline negative & 48 & $36(50.2)$ & $12(30.8)$ & \multirow{5}{*}{0.002} \\
\hline positive & 52 & $25(49.8)$ & $27(69.2)$ & \\
\hline Preoperative URS & & & & \\
\hline not performed & 53 & $40(65.6)$ & $13(33.3)$ & \\
\hline performed & 47 & $21(34.4)$ & $26(66.7)$ & \\
\hline
\end{tabular}

$\mathrm{IVR}=$ intravesical recurrence; $\mathrm{CIS}=$ carcinoma in situ.

\subsection{The Association Between Surgical Outcome Factors and IVR}

Though, the total operation time was 370 min (206 to 570) in the non-IVR group and $396 \mathrm{~min}$ (238 to 690) in the IVR group, with no significant association $(\mathrm{P}=0.25)$, the pneumoperitoneum time was $197 \mathrm{~min}$ (95-390) in the non-IVR group and $237 \mathrm{~min}(120-390)$ in the IVR group, with significant difference $(\mathrm{P}=0.0005)$.

\subsection{The Association Between Pathological Factors and IVR Rate}

Preoperative urine cytology was highly significant association for IVR $(<0.0001)$. The tumor size was $27 \mathrm{~mm}$ in the non-IVR group and $39 \mathrm{~mm}$ in the IVR group, with significant difference $(\mathrm{P}=0.001)$. Furthermore, the $\mathrm{pT}$ stage was $34(55.7 \%)$ for equal or lower than pT1, $16(26.2 \%)$ for pT2, and $11(18 \%)$ for equal or higher than pT3 in non-IVR group, and respectively $13(33.3 \%), 10(25.6 \%), 16(41 \%)$ in IVR group, with significant difference $(\mathrm{P}=0.01)$. No significant difference was observed with regard to concomitant CIS, vascular invasion, lymphovascular invasion, pathological $\mathrm{N}$ stage, and resected margin.

\subsection{The Association Between Postoperative Therapy and IVR Rate}

Adjuvant chemotherapy was performed $30(49 \%)$ patients in the non-IVR group and $10(25.6 \%)$ patients in the IVR group, with significant association $(\mathrm{P}=0.019)$.

\subsection{IVR Free Survival (IVRFS) According to Respectively Factors}

We further examined the impact of clinico-pathological factors on IVRFS (Figure 2). The Kaplan-Meier Curves showed that the IVRFS estimates after LRNU were $72 \%$ for the renal pelvic cancer compared with $52 \%$ for the ureteric cancer $(P=.003), 52 \%$ for positive smoking history compared with $68 \%$ for negative smoking history $(P=.004)$, $48 \%$ for positive hydronephrosis compared with $75 \%$ for negative hydronephrosis $(P=.003), 40 \%$ for positive urine cytology compared with $79 \%$ for negative urine cytology $(P<.0001), 45 \%$ for tumor $>3 \mathrm{~cm}$ compared with $83 \%$ for 
tumor $\leq 3 \mathrm{~cm}(P=.001), 44 \%$ for underwent URS compared with $75 \%$ for not underwent URS $(P=.006)$, $48 \%$ for pneumoperitoneum time $>200$ minutes compared with $83 \%$ for pneumoperitoneum time $\leq 200$ minutes $(P=.002)$, and

(A) Tumor location

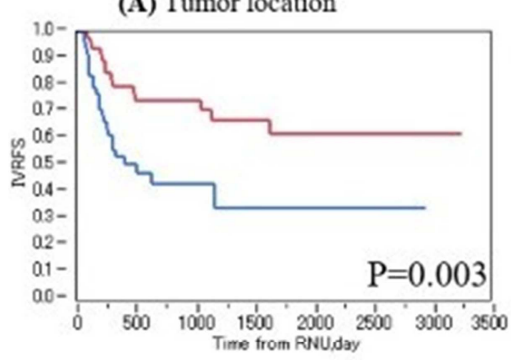

- :renal pelvis

$$
\text { - ureter }
$$

(C) Preoperative cytology
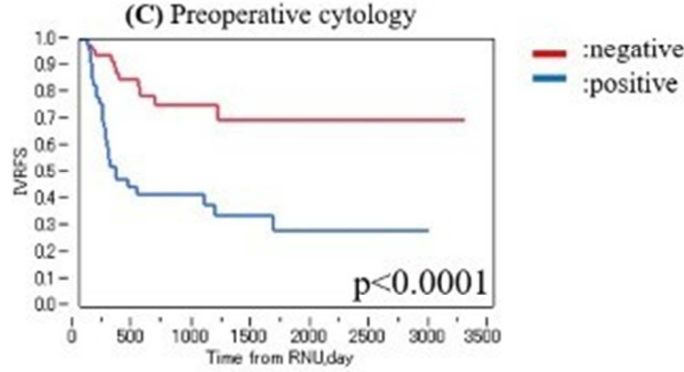

(E) Blickmann index
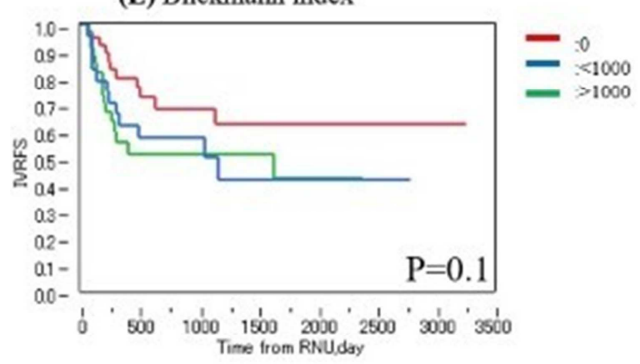

(G) URS

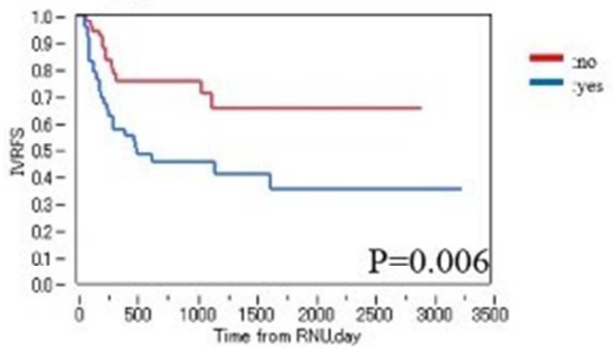

(I) Pneumoperitoneum time

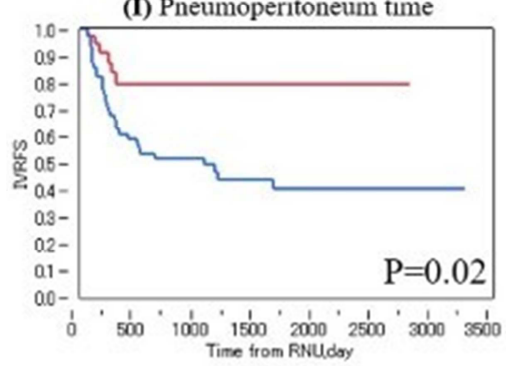

$75 \%$ for performed Adjuvant chemotherapy compared with $52 \%$ for not performed Adjuvant chemotherapy $(P=.001)$. However, Blickmann Index did not show statistically significant differences with respect to IVRFS.

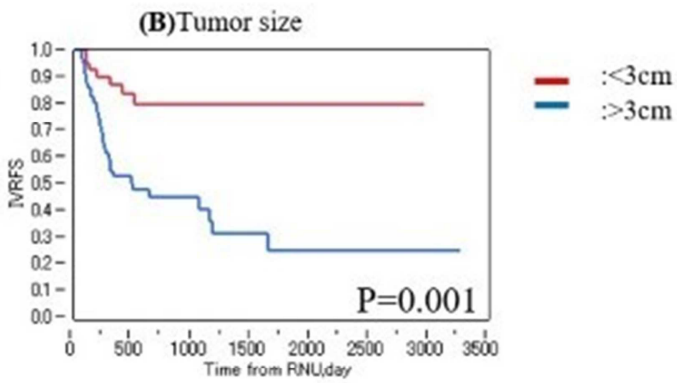

(D) Smoking history

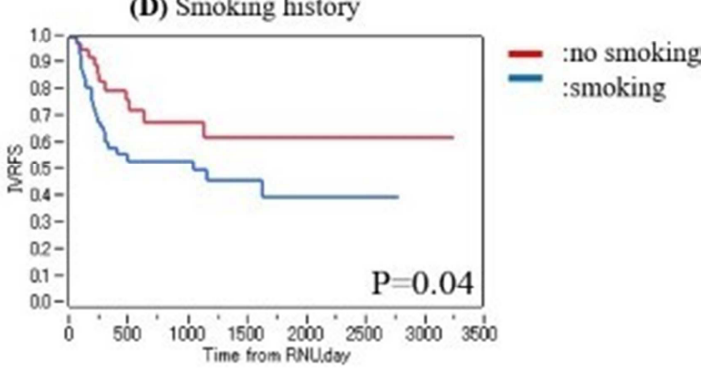

(F) Hydronephrosis

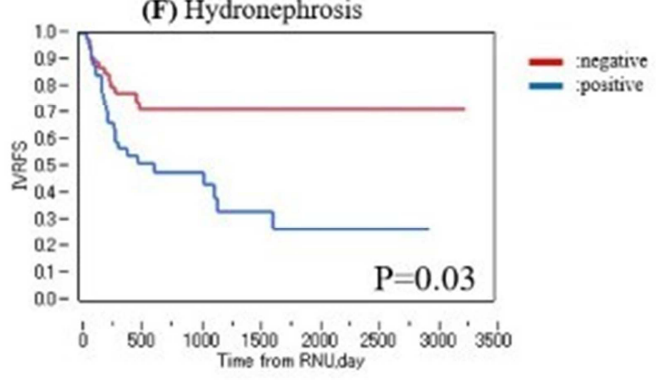

(H) pT

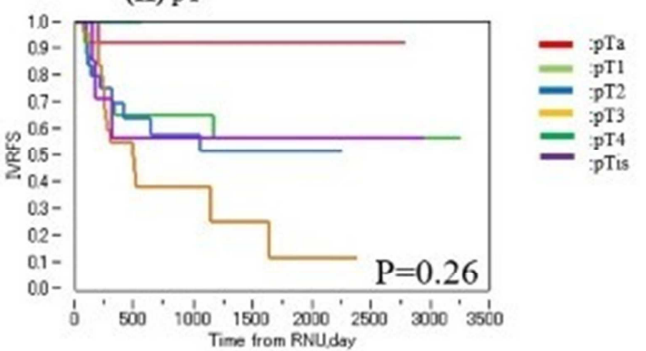

(J) Ajuvant chemotherapy

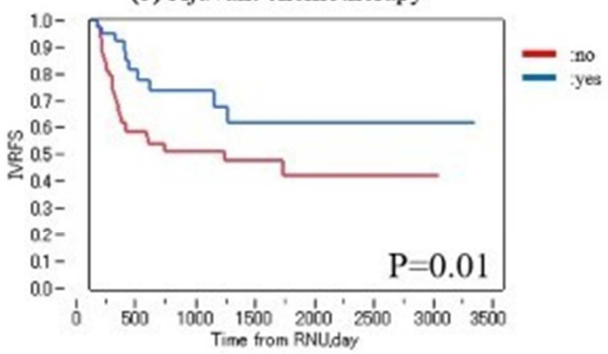

Figure 2. Kaplan-Meier Curves of the IVRFS stratified by tumor location (A), tumor size (B), preoperative urinary cytology (C), smoking history (D), and Blickmann index $(E)$, hydronephrosis $(F)$, receipt of URS before $L R N U(G)$, pathologic $T$ stage $(H)$, pneumoperitoneum time (I), and receipt of ajuvant chemotherapy $(J)$. 
Univariate analysis showed that tumor location (HR, 2.6; 95\% CI, 1.358-5.193; $P=.004$ ), tumor size (HR, 2.075; 95\% CI, 1.979-8.152; $P=.02)$, positive preoperative urinary cytology (HR, 3.87; 95\% CI, 1.979-8.152; $P<.0001$ ), hydronephrosis (HR, 2.579; 95\% CI, 1.332-5.299; $P=.005$ ), URS before LRNU (HR, 2.425; 95\% CI, 1.268-4.872; $P=.007$ ), pneumoperitoneum time (HR, 2.87; 95\% CI,
1.289-7.618; $P=.008)$, smoking history (HR, 1.967; 95\% CI, 1.007-4.130; $P=.04$ ), and Blickmann index (HR, 1.938; 95\% CI, 1.028-3.745; $P=.04)$, were poor prognostic factors for IVR. However, Adjuvant chemotherapy (HR, 0.458; 95\% CI, $0.212-0.909 ; P=.003)$ was decreased the probability of IVR (Table 3).

Table 3. Univariate and Multivariate Cox Regression Analyses Predicting IVR in 100 Patients Who Underwent LRNU for UTUC.

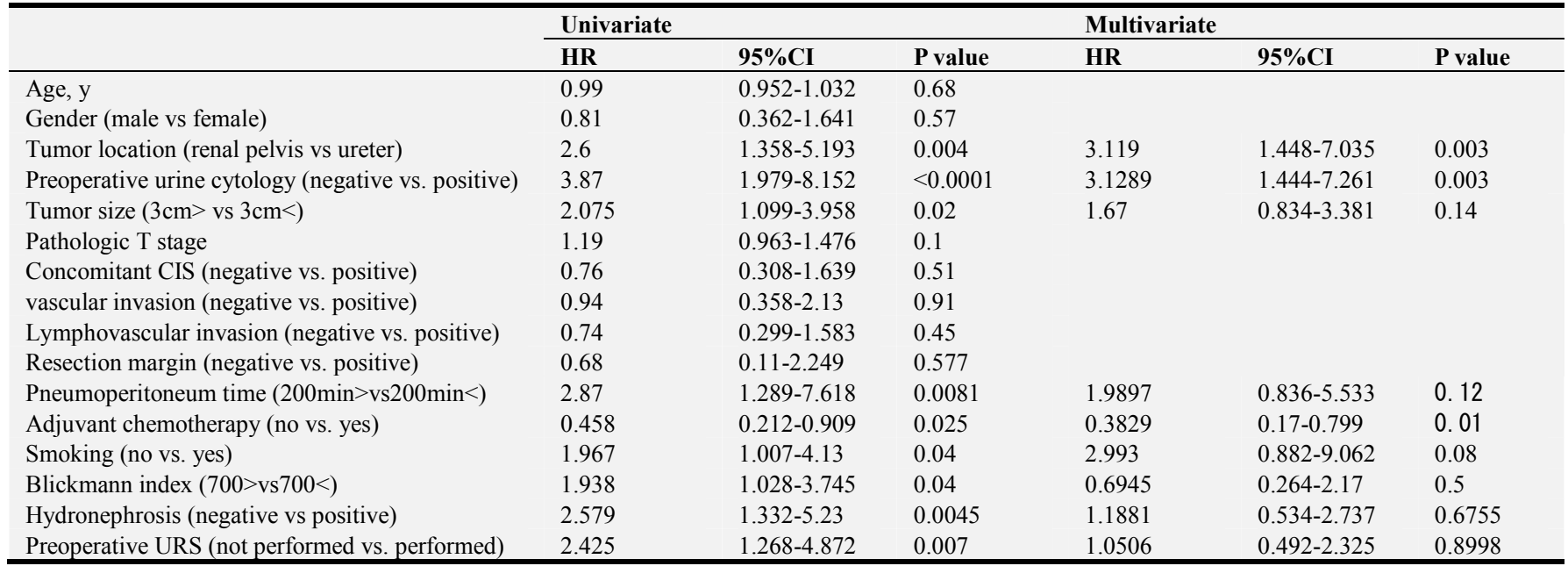

Multivariate analysis also showed that tumor location (HR, $3.12 ; 95 \%$ CI, $1.448-7.035 ; \quad P=.0003)$ and positive preoperative urinary cytology (HR, 3.128; 95\% CI, 1.444-7.261; $P=.003)$, were independent risk factors of IVR. However, enforcement of Adjuvant chemotherapy (HR, $0.382 ; 95 \% \mathrm{CI}, 0.170-0.799 ; \mathrm{P}=.001$ ) was associated with a significant reduction in the risk of IVR.

We further examined the impact of IVR on overall survival (OS). 28 (28\%) patients died. The median duration of OS was 33 months (range, 1-118.5). No significant association of a IVR with OS $(\mathrm{p}=0.48)$ was observed in the Kaplan-Meier Curves of OS (Figure 3).

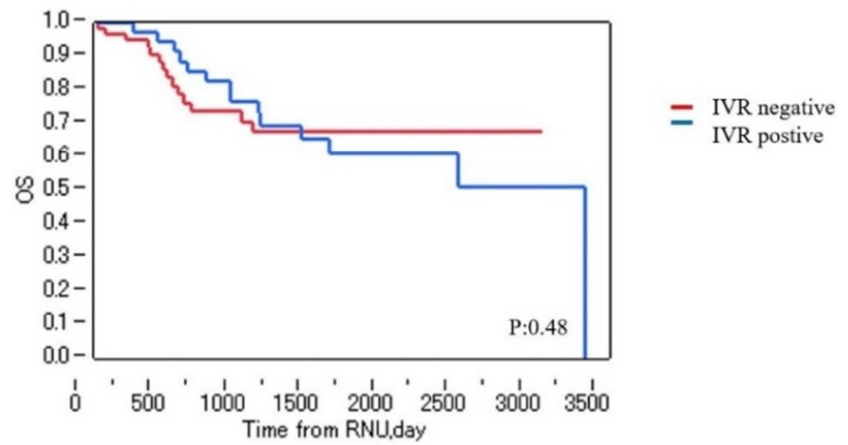

Figure 3. Kaplan-Meier Survival Curves of the overall survival stratified according to IVR status. there is no significant difference between positive IVR and negative IVR.

\section{Discussion}

In recent years, laparoscopic surgery can be safely performed even in RNU with the development of laparoscopic techniques. Postoperative IVR is relatively frequent at approximately 15 to $50 \%$ after RNU [5]. The previous study showed the several risk factors for IVR, including the laparoscopic procedure [3]. In our study, we found that $34.3 \%$ of patients with UTUC experienced IVR within a median interval of 13.6 months between RNU and the first IVR. However, LRNU was not significantly more frequent more than in ORNU $(\mathrm{P}=0.36)$. In LRNU patients, the ureteral cancer was an independent risk factor for IVR compare with renal pelvic cancer $(\mathrm{P}=0.0003)$. In particular, the tendency was more strong influence in the lower ureter. Under laparoscopic procedure, the clamping of ureter below the tumor is difficult, thus there is a possibility that a large amount of cancer cells may flow into the bladder. In addition, Tumor size $>3 \mathrm{~cm}$ was a risk factor for IVR in our study (univariate analysis, $\mathrm{P}=0.02$ ). The recent study has shown that tumor size was significantly associated with IVR [6]. The previous studies describing the prognostic factor of positive preoperative urinary cytology [7, 8]. In our study, the similar results were obtained. Furthermore, we obtained the result that preoperative hydronephrosis was significantly associated for IVR $(\mathrm{P}=0.007)$. Previous multicenter analysis described that presence of the hydronephrosis had multifocal tumors and positive urinary cytology (all $P<.001$ ), and these factors may be responsible for the increased rate of IVR [6]. We think that the presence of hydronephrosis is likely to pass through the urinary tract, many cancer cells may fall in the bladder and may cause increasing IVR. We found that preoperative URS increased the risk of IVR $(\mathrm{P}=0.002)$. The Cancer cell transplantation during URS can be explained as a cause of increased IVR. Previous studies also reported 
similar results for the effect of preoperative URS on IVR [9, 10]. In the large tumor, we could easily diagnose UTUC by contrast-enhanced CT scan. Furthermore, we recently performed PET-CT scan with diuretic drug for small size tumor and we have to be able to relatively easily diagnosed. As a result, the patients who underwent preoperative URS tend to decrease and LRNU was performed without preoperative URS to prevent the delay of definitive surgical treatment. Prospective studies are needed to clarify increased positive urine cytology in bladder urine sample after URS. We demonstrated that positive smoking history and higher Blickmann index were significant risk factor for IVR $(\mathrm{P}=0.03$ and 0.01). Smoking are generally at risk for developing bladder cancer, we think that it can also be a risk factor for IVR after the LRNU. In the group who received postoperative chemotherapy, we showed that the frequency of IVR decreased (multivariate analysis, HR: 0.3829, 95\%CI: 0.17-0.799, $\mathrm{P}=0.01$ ) and this result suggested that adjuvant systemic chemotherapy had an effect of preventing IVR. Chan Ho Lee et al also found that the adjuvant systemic chemotherapy decreased the risk of IVR [6]. Soga et al and $\mathrm{Ku}$ et al indicated that the reduction of IVR risk with adjuvant chemotherapy. On the other hand, a study from Canadian Upper Tract Collaboration indicated that the increase of IVR risk with adjuvant chemotherapy [11-13]. We generally apply and administer an adjuvant systemic chemotherapy to more pathological T1 case or positive vascular invasion case. In general, postoperative chemotherapy is performed for the purpose of preventing local recurrence or distant metastasis, as a result, the effect to reduce IVR risk was also suggested. In this study, there is no significance IVR rate between ORNU and LRNU. However, we found that extension of pneumoperitoneum time in LRNU was significant risk factor for IVR $(\mathrm{P}=.0005)$. Previous studies also reported similar results for the risk of extension of pneumoperitoneum time on IVR [14]. Several studies have pointed out that the procedure-related elevated-pressure pneumoperitoneum may causes the gravitational migration of tumor cells, which leads to tumor cell seeding to the bladder $[1,15,16]$. Prolonged pneumoperitoneum time under the LRNU may be associated with higher rate IVR [17]. In this reason, we hypothesized that one is $\mathrm{CO}_{2}$ gas high pressure was associated with excitation of the sympathetic nervous system and it coursed the promotion of peristaltic movement of the ureter. In our institution, we normally perform laparoscopic surgery by $\mathrm{CO}_{2}$ gas pressure setting $8 \mathrm{cmHg}$ (12 $\mathrm{cmHg}$ at the time of bleeding). The early ligation of the ureter distal to the tumor site during RNU has been suggested to avoid tumor spillage into the bladder $[18,19]$. However, lower ureteral tumor case is difficult to clamping of the ureter distal to the tumor site duaring laparoscopic procedure. In our study, the lower ureteric tumor patients were significant higher IVR rate than renal pelvic and upper ureteral tumor. This result suggested that early clamping of distal ureter was important procedure for preventing IVR. On the other hand, the previous study showed that clamping the ureter before the ligation of renal vessels had a worse impact on CSS. They ultimately concluded that early ureteral ligation may cause a rapid increase of intrarenal-pelvic pressure, which results in the spread and dispersal of cancer cells flowing back into the blood and perinephric space [20]. Therefore, early ureteral clamping may be decrease postoperative IVR rate, but this procedure may be increased postoperative hematogenous metastasis. We think that it is important to keep more lower $\mathrm{CO}_{2}$ gas pressure, shorten the pneumoperitoneum time, earlier clamping of the ureter distal to the tumor site and supportive laparoscopic procedure. As a matter of course, we think that it is important to check the presence of IVR regularly after LRNU, but we also consider the follow-up of metastasis. We demonstrated that the independent predictive risk factor for IVR were ureteral tumor (lower) and positive preoperative urine cytology. High risk patients who has these risk factors are needed the perioperative intravesical therapy such as intravesical instillation of pirarubicin or adjuvant systemic chemotherapy. Several studies had been reported that immediately intravesical instillation therapy after RNU is effective for prevention of IVR. In a multicenter randomized study, the intravesical instillation of mitomycinC after RNU was significantly suppressed the IVR [21]. In Japanease randomized trial study, the IVR prevention effect of single intravesical instillation of pirarubicin was significantly suppressed compare with control group [22].

We report a study of IVR after LRNU in our department. Though, the previous clinical studies showed that postoperative IVR were significantly higher in laparoscopic surgery, there was not significant difference between LRNU and ORNU in our study. We were showed that the independent predictive risk factor for IVR were lower ureteral tumor and positive preoperative urine cytology, and adjuvant systemic chemotherapy reduced the risk of IVR. In this study, we suggest that high risk patients who has these risk factors are needed the perioperative intravesical therapy and/or adjuvant systemic chemotherapy for the prevention of IVR.

\section{Conflict of Interest}

None declared.

\section{References}

[1] E. Xylinas, P. Colin, F. Audenet, et al. Intravesical recurrence after radical nephroureterectomy for upper tract urothelial carcinomas: predictors and impact on subsequent oncological outcomes from a national multicenter study, World J. Urol. 2013; 31: 61-68.

[2] E. Xylinas, L. Kluth, N. Passoni, et al. Prediction of intravesical recurrence after radical nephroureterectomv: development of a clinical decision-making tool, Eur. Urol. 2014; 65: 650-658.

[3] Thomas Seisen, Benjamin Granger, et al. A Systematic Review and Meta-analysis of Clinicopathologic Factors Linked to Intravesical Recurrence after Radical Nephroureterectomy to Treat Upper Tract Urothelial Carcinoma. Eur Urol. 2015 Jun; 67 (6): 1122-1133. 
[4] V. Margulis, S. F. Shariat, S. F. Matin, et al. Outcomes of radical nephroureterectomy: a series from the upper tract urothelial carcinoma collaboration, Cancer 2009; 115: 1224-1233.

[5] Azémar MD, Comperat E,, Richard F, Cussenot O, Rouprêt M. Bladder recurrence after surgery for upper urinary tract urothelial cell carcinoma: fre- quency, risk factors, and surveillance. Urol Oncol. 2011; 29: 130-6.

[6] Chan Ho Lee, Ja Yoon Ku, Chang Wook Jeong, Ja Hyeon Ku, Predictors for Intravesical Recurrence Following Radical Nephroureterectomy for Upper Tract Urothelial Carcinoma: A National Multicenter Analysis. Clinical Genitourinary Cancer, 2017; 15: 1055-61.

[7] Tanaka N, Kikuchi E, Kanao K, et al. The predictive value of positive urine cytology for outcomes following radical nephroureterectomy in patients with primary upper tract urothelial carcinoma: a multi-institutional study. Urol Oncol 2014; 32 (48): 19-26.

[8] Kobayashi Y, Saika T, Miyaji Y, et al. Preoperative positive urine cytology is a risk factor for subsequent development of bladder cancer after nephroureterectomy in patients with upper urinary tract urothelial carcinoma. World J Urol 2012; 30: $271-5$.

[9] Luo HL, Kang CH, Chen YT, et al. Diagnostic ureteroscopy independently correlates with intravesical recurrence after nephroureterectomy for upper urinary tract urothelial carcinoma. Ann Surg Oncol 2013; 20: 3121-6.

[10] Sankin A, Tin AL, Mano R, et al. Impact of ureteroscopy before nephroureterectomy for upper tract urothelial carcinoma on oncologic outcomes. Urology 2016; 94: 148-53.

[11] Fradet V, Mauermann J, Kassouf W, et al. Risk factors for bladder cancer recurrence after nephroureterectomy for upper tract urothelial tumors: results from the Canadian Upper Tract Collaboration. Urol Oncol 2014; 32: 839-45.

[12] Ku JH, Choi WS, Kwak C, Kim HH. Bladder cancer after nephroureterectomy in patients with urothelial carcinoma of the upper urinary tract. Urol Oncol 2011; 29: 383-7.

[13] Soga N, Arima K, Sugimura Y. Adjuvant methotrexate, vinblastine, adriamycin, and cisplatin chemotherapy has potential to prevent recurrence of bladder tumors after surgical removal of upper urinary tract transitional cell carcinoma. Int J Urol 2008; 15: 800-3.
[14] Keisuke Shigeta, Eiji Kikuchi, Masayuki Hagiwara, Toshiyuki Ando, Ryuichi Mizuno. Prolonged pneumoperitoneum time is an independent risk factor for intravesical recurrence after laparoscopic radical nephroureterectomy in upper tract urothelial carcinoma. Surgical Oncology 2017; 26: 73-79.

[15] V. Fradet, J. Mauermann, W. Kassouf, et al. Risk factors for bladder cancer recurrence after nephroureterectomy for upper tract urothelial tumors: results from the Canadian Upper Tract Collaboration, Urol. Oncol. 2014; 32: 839-845.

[16] L. Zou, L. Zhang, H. Zhang, H. Jiang, Q. Ding. Comparison of post-operativeintravesical recurrence and oncological outcomes after open versus laparoscopic nephroureterectomy for upper urinary tract urothelial carcinoma, World J. Urol. 2014; 32: 565-570.

[17] Eng MK, Shalhav AL. Laparoscopic nephroureterectomy: long-term outcomes. Curr Opin Urol. 2008; 18: 157-62.

[18] Zigeuner RE, Hutterer G, Chromecki T, Rehak P, Langner C. Bladder tumour development after urothelial carcinoma of the upper urinary tract is related to primary tumour location. $B J U$ Int 2006; 98: 1181-6.

[19] Kurzer E, Leveillee RJ, Bird VG. Combining hand assisted laparoscopic nephroureterectomy with cystoscopic circumferential excision of the distal ureter without primary closure of the bladder cuff-is it safe? J Urol 2006; 175: 63-7, discussion: 67-8.

[20] J. Inokuchi, K. Kuroiwa, S. Naito, et al. The impact of ureteral ligation on clinical outcome during radical nephroureterectomy for upper tract urothelial carcinoma: multi-institutional case series study JCOG 1110A, Eur. Urol. Suppl. 2016; 15: 801.

[21] O'Brien T, Ray E, Singh R, Coker B, Beard R; British Association of Urological Surgeons Section of Oncology. Prevention of bladder tumours after nephroureterectomy for primary upper urinary tract urothelial carcinoma: a prospective, multicentre, randomised clinical trial of a single postoperative intravesical dose of mitomycin $\mathrm{C}$ ( the ODMIT-C Trial） . Eur Urol. 2011; 60: 703-10.

[22] Ito A, Shintaku I, Satoh M, et al. Prospective randomized phase II trial of a single early intravesical instillation of pirarubicin (THP) in the prevention of bladder recurrence after nephroureterectomy for upper urinary tract urothelial carcinoma: the THP Monotherapy Study Group Trial. J Clin Oncol. 2013; 31: 1422-7. 